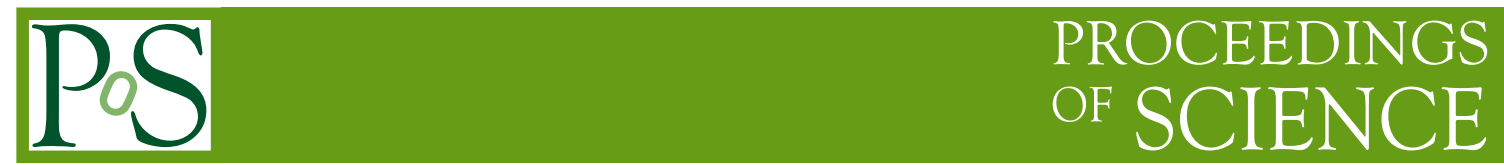

\title{
XYZ States
}

\section{Wei Chen}

Department of Physics, Peking University, China

and Department of Physics and Engineering Physics, University of Saskatchewan, Canada

\section{Wei-Zhen Deng}

Department of Physics, Peking University, China

\section{Jun He}

Nuclear Theory Group, Institute of Modern Physics of CAS, Lanzhou 730000, China

\section{Ning Li}

Department of Physics, Peking University, China

and Institut für Kernphysik and Jülich Center for Hadron Physics, Forschungszentrum Jülich

\section{Xiang Liu}

School of Physical Science and Technology, Lanzhou University, Lanzhou 730000, China

\section{Zhi-Gang Luo}

Department of Physics, Peking University,

and Department of Physics, Hubei Institute of Technology

\section{Zhi-Feng Sun}

School of Physical Science and Technology, Lanzhou University, Lanzhou 730000, China

\section{Shi-Lin Zhu ${ }^{* \dagger}$}

Department of Physics and State Key Laboratory of Nuclear Physics and Technology and Collaborative Innovation Center of Quantum Matter,

Peking University, Beijing 100871, China

E-mail: zhuslepku.edu.cn

In the past decade, many new charmonium (or charmonium-like) and bottomonium (or bottomonium-like) states were observed experimentally. I will review these XYZ states which do not fit into the quark model spectrum easily.

XV International Conference on Hadron Spectroscopy-Hadron 2013

4-8 November 2013

Nara, Japan

\footnotetext{
* Speaker.

${ }^{\dagger}$ Plenary talk.
} 


\section{The Charmonium Spectrum}

In the past decade many new charmonium and Upsilon (or charmonium-like and bottomoniumlike) states were observed experimentally. Their production mechanisms include the initial state radiation (ISR), double charmonium production, two photon fusion, B decays and excited charmonium or bottomonium decays. These new states were observed through either the hiddencharm/hidden-bottom or open-charm/open-bottom final states.

Up to now the lattice QCD simulation reproduced the charmonium spectrum below the $D \bar{D}$ threshold very well. However, many new states above the $D \bar{D}$ threshold were observed since 2003. Some are very narrow or even charged, which are good candidates of exotic mesons. Beside the conventional $q \bar{q}$ mesons and $q q q$ baryons, QCD allows many other possible color singlets such as dibaryons, pentaquarks, glueballs, tetraquarks, hadronic molecules, hybrid hadrons etc.

Many new charmonium or charmonium-like states do not fit into the quark model spectrum easily. Theoretical speculations include: di-meson molecular states, tetraquarks, hybrid charmonium and conventional charmonium. Molecular states are loosely bound states composed of a pair of heavy mesons. They are probably bound by the long-range color-singlet pion exchange. Tetraquarks are bound states of four quarks, which are bound by colored force between quarks. They decay through rearrangement. There are many states within the same tetraquark multiplet. Some members are charged or carry strangeness. Hybrid charmonium are bound states composed of a pair of quarks and one excited gluon. Last but not the least, these new states could still be conventional charmonium. The quark model spectrum could be distorted by the coupled-channel effects.

Especially many charmonium-like states lie very close to the open-charm threshold. Are these threshold enhancements real resonances? Could they arise from the cusp effect, final state interaction, interference between continuum and well-known charmonium states or triangle singularity due to the special kinematics? These possibilities always exist. One should be very cautious in order to establish that a threshold enhancement is a genuine resonance.

\section{The Vector Charmonium Family}

In the quark model, one expects at most five vector charmonium states between 4 and $4.7 \mathrm{GeV}$ : $3 S / \psi(4040), 2 D / \psi(4160), 4 S / \psi(4415), 3 D, 5 S$. But seven states were observed experimentally: $\psi(4008), \psi(4040), \psi(4160), \psi(4260), \psi(4360), \psi(4415), \psi(4660)$. What are these additional $\mathrm{Y}$ states?

Y(4260) was first discovered in the $J / \psi \pi \pi$ mode with the ISR technique by Babar collaboration and confirmed by Belle and CLEO collaboration. Y(4360) was observed in the $\psi(2 S) \pi \pi$ channel with ISR by Babar collaboration. Up to now there has been no evidence of these two states in the open-charm process and R-value scan. As mentioned earlier, there exist some possible nonresonant interpretations of these states. For example, one may introduce the interference between the continuum and the well established resonances such as $\psi(4160)$ and $\psi(4415)$ to reproduce the line shape of the $J / \psi \pi \pi$ and $\psi(2 S) \pi \pi$ spectrum quite well [1].

It's quite possible that $\mathrm{Y}(4260)$ may be a conventional charmonium. The bare $c \bar{c}$ state in the quark model may mix with the $D^{(*)} \bar{D}^{(*)}$ continuum through the $D^{(*)} \bar{D}^{(*)}$ hadron loop. The char- 
monium spectrum might be distorted. For example, the screened linear potential was introduced to model the correction from the light quark pair creation in the vacuum (or the correction from the hadron loop). The energy level spacing above $4 \mathrm{GeV}$ becomes narrower. More vector states can exist between 4 and $4.7 \mathrm{GeV}$. With such a scheme, Y(4260) can be regarded as the $\psi(4 S)$ charmonium state [2].

Could some Y states be tetraquarks? According to the QCD sum rule analysis, the hiddencharm vector states lie around $4.6 \mathrm{GeV}$ [3]. However, the hidden-charm tetraquarks will fall apart into a pair of open-charm D mesons or hidden-charm plus light mesons very easily. Their width is expected to be very large while $\mathrm{Y}$ states are not so broad. Up to now, $\mathrm{Y}$ states have not been observed in the p-wave $D^{(*)} \bar{D}^{(*)}$ modes.

If it's a genuine resonance, $\mathrm{Y}(4260)$ is a very good candidate of the charmonium hybrid [4, 5, 6]. According to lattice QCD simulation [7], both the vector $\left(1^{--}\right)$and exotic $\left(1^{-+}\right)$hybrid charmonium lie around $4.26 \mathrm{GeV}$. One naively expects that the vector hybrid charmonium does not couple to the virtual photon very strongly due to the intrinsic gluon, which leads to the dip in the $\mathrm{R}$ value scan. According to the flux tube model and QCD sum rule analysis [8], the favorable decay mode of hybrid states is the p-wave + s-wave meson pair, which explains the non-observation in the $D^{(*)} \bar{D}^{(*)}$ modes. The $c \bar{c}$ pair within the vector charmonium is a spin-singlet, which is favorable to the spin-singlet hidden-charm decay mode. Recently BESIII collaboration observed Y(4260) in the $h_{c} \pi \pi$ and p-wave + s-wave $\mathrm{D}$ meson decay modes.

\section{The Charged States And X(3872)}

Belle collaboration reported two charged states $Z_{b}(10610)$ and $Z_{b}(10650)$ in 2011 [9]. This year BESIII collaboration observed two charged $Z_{c}$ states [10, 11]. The lower state $Z_{c}(3900)$ was confirmed by Belle [12] and Cleo collaborations [13] very quickly.

The two $Z_{c}$ and $Z_{b}$ states are very similar. They are charged charmonium-like (or bottomoniumlike) structures close to the open-charm (or open-bottom) threshold. Their quantum numbers are exactly the same with $I^{G} J^{P}=1^{+} 1^{+}$. They were observed both in the hidden-charm (or hiddenbottom) and open-charm (or open-bottom) final states. Moreover, the open-charm (or open-bottom) modes dominate the $Z_{c}$ (or $Z_{b}$ ) decays.

Could $Z_{c}$ (or $Z_{b}$ ) be tetraquarks? The isovector and axial vector hidden-charm tetraquarks do lie around $4 \mathrm{GeV}$ as shown in Table V in Ref. [3]. However, the hidden-charm tetraquarks will fall apart into a pair of open-charm D mesons or one charmonium plus light mesons very easily. Their width is expected to be large while the $Z_{c}$ (or $Z_{b}$ ) states are very narrow experimentally. Moreover, the s-wave $\bar{D} D^{*}\left(\bar{B} B^{*}\right)$ mode should dominate the $\bar{D}^{*} D^{*}$ (or $\bar{B}^{*} B^{*}$ ) mode because of the large phase space difference. For example, the phase space of the open-charm decay $Z_{c}(4020) \rightarrow \bar{D}^{*} D^{*}$ is tiny and strongly suppressed compared to that of $Z_{c}(4020) \rightarrow \bar{D} D^{*}$. Experimentally, the higher $Z_{b}(10650)$ state was not observed in the s-wave $\bar{B} B^{*}$ mode while the higher $Z_{c}(4020)$ has not been observed in the s-wave $\bar{D} D^{*}$ mode. Therefore, $Z_{c}$ and $Z_{b}$ seems not good candidates of the hiddencharm/bottom tetraquarks unless one can invent some particular dynamics which forbids the $\bar{D} D^{*}$ and $\bar{B} B^{*}$ decay modes.

In fact, there exists a very natural interpretation. $Z_{b}$ states can be regarded as candidates of the $S$-wave $\bar{B} B^{*}$ and $\bar{B}^{*} B^{*}$ molecular states $[14,15,16]$. Similarly, $Z_{c}$ states are candidates of the 
S-wave $\bar{D} D^{*}$ and $\bar{D}^{*} D^{*}$ molecular states or molecular-type resonances [17, 18]. There are many literatures along this direction [19, 20, 21].

In QED we have the hydrogen atom where the light electron circles around the proton. We also have the hydrogen molecule where two electrons shared by two protons. In QCD, we have the heavy meson where the light quark circles around heavy quark. We may also expect the di-meson molecule where the two mesons are bound by the pion exchange force.

The idea of the loosely bound molecular states is not new in nuclear physics since Yukawa proposed the pion in 1935. The deuteron is a very loosely bound state composed of a proton and neutron arising from the color-singlet meson exchange. Besides the long-range pion exchange, the medium-range attraction from the correlated two-pion exchange (or in the form of the sigma meson exchange), the short-range interaction in terms of the vector meson exchange, and the S-D wave mixing combine to form the loosely bound deuteron. The deuteron is a perfect hadronic molecular state!

We adopt the same one-boson-exchange formalism to discuss the possible molecular states composed of a pair of heavy mesons. Within this framework, both $Z_{b}(10610)$ and $Z_{b}(10650)$ can be explained as the $\mathrm{S}$-wave $\bar{B} B^{*}$ and $\bar{B}^{*} B^{*}$ molecular states. Besides the isovector $Z_{b}$ states, there are also several loosely bound isoscalar molecular states [14]. The same analysis holds for the $Z_{c}$ system [17, 18].

Then how about X(3872)? In Refs. [22, 15, 16], we considered (i) the S-D wave mixing which plays an important role in forming the loosely bound deuteron; (ii) both the neutral $\bar{D}^{0} D^{* 0}$ and charged $\bar{D}^{+} D^{*-}$ component in the flavor wave function; (iii) the mass difference between the neutral and charged $\bar{D} D^{*}$ meson; and (iv) the coupling of $\bar{D} D^{*}$ to $\bar{D}^{*} D^{*}$ channel. It turns out that $\mathrm{X}(3872)$ is a very loosely bound molecular state. When the binding energy is $0.3 \mathrm{MeV}$, the branching fraction ratio between the isospin conserving $J / \psi \pi^{+} \pi^{-} \pi^{0}$ mode and isospin violating $J / \psi \pi^{+} \pi^{-}$is 0.42 [22], which agrees well with both Babar's measurement $0.8 \pm 0.3$ and Belle's measurement $1.0 \pm 0.4 \pm 0.3$. It's important to note that we do not need to add by hand the $J / \psi \rho$ and $J / \psi \omega$ component into the flavor wave function of $\mathrm{X}(3872)$ in order to explain the large isospin violation in its strong decays.

The existence of the loosely bound state X(3872) and the large isospin symmetry breaking in its hidden-charm decay arises from the very delicate efforts of the several driving forces including: the long-range one-pion exchange, S-D wave mixing, mass splitting between the charged and neutral $D\left(D^{*}\right)$ mesons and coupled-channel effects. The extreme sensitivity of the physical observables to the tiny binding energy is typical of the loosely bound system.

Then how can we further test the one-pion-exchange model and molecular picture? We can test the molecular picture through the isoscalar partner of $Z_{c}(3900)$, isovector partner states in the $Z_{c}(4020)$ multiplet with $I^{G}\left(J^{P}\right)=1^{-}\left(0^{+}\right), 1^{+}\left(1^{+}\right), 1^{-}\left(2^{+}\right)$, and isoscalar partner states of $Z_{c}(4200)$ with $I^{G} J^{P C}=0^{+}\left(0^{++}\right), 0^{-}\left(1^{+-}\right), 0^{+}\left(2^{++}\right)$. One may wonder whether $\mathrm{X}(3872)$ is the isoscalar partner of $Z_{c}(3900)$. We can also investigate the pionic, di-pion and electromagnetic decay pattern of the $Z_{c}(3900)$ and $Z_{c}(4020)$ multiplet with the heavy quark spin and flavor symmetry. Details can be found in Ref. [17]. Similar discussions hold for the $Z_{b}$ states. 


\section{The Possible Landscape of Hadronic Molecules}

This year BESIII collaboration reported the hidden-charm decays $Y(4260) \rightarrow \pi Z_{c}(3900,4020)$, $Y(4360) \rightarrow \pi Z_{c}(3900,4020)$ and radiative decay $Y(4260) \rightarrow \gamma X(3872)$ with a large ratio $\frac{\Gamma[\gamma X(3872)]}{\Gamma[\pi \pi J / \psi]}=$ $11.2 \%$ [23]. It seems that there might exist some possible connections between various XYZ states. Are they related to each other? Could they have the same inner structure?

Theoretical speculations of Y(4260) include (a) $\psi(4 S)$; (b) a vector hybrid charmonium; (c) a $D_{1} \bar{D}$ molecule. In contrast, $\mathrm{X}(3872)$ could be (1) $\chi_{c 1}^{\prime}$; (2) a $\bar{D} D^{*}$ molecule; (3) the mixture of $\chi_{c 1}^{\prime}$ and $\bar{D} D^{*}$ molecule. There are many combinations. In the following let's discuss several typical scenarios.

- *[Scenario 1:] $\mathrm{Y}(4260)=\psi(4 S)$ and $\mathrm{X}(3872)=\chi_{c 1}^{\prime}$. Then we expect no more charged partner states around $\mathrm{Y}(4260)$ ? It's very challenging to explain why Y(4260) was not observed in p-wave $\bar{D}^{(*)} D^{(*)}$ modes? It's also very difficult to explain the large $J / \psi \pi \pi$ branching ratio of $\mathrm{Y}(4260)$ ? For comparison, the $J / \psi \pi \pi$ branching ratio of well established excited charmonium is around $1 \%$. Moreover, where is the isoscalar partner of $Z_{c}(3900)$ if it's not an experimental fake signal?

- *[Scenario 2:] $\mathrm{Y}(4260)=\psi(4 S)$ and $\mathrm{X}(3872)=\bar{D} D^{*}$ molecule. Again, the same puzzle of $\mathrm{Y}(4260)$ exists as in Scenario 1. Then where is $\chi_{c 1}^{\prime}$ ?

- *[Scenario 3:] $\mathrm{Y}(4260)=$ hybrid charmonium and $\mathrm{X}(3872)=\chi_{c 1}^{\prime}$. There are no more charged partner states around $\mathrm{Y}(4260)$. Then what is the similar state $\mathrm{Y}(4360)$ ? Where is the isoscalar partner of $Z_{c}(3900)$ ?

- *[Scenario 4:] $\mathrm{Y}(4260)=D_{1} \bar{D}$ molecule and $\mathrm{X}(3872)=\bar{D} D^{*}$ molecule. This scenario is both interesting and a little wild. There will be many charged partner states around Y(4260). Y(4360) may also be a molecular state. With Scenario 4, we expect a landscape of hadronic molecules.

First we consider molecules composed of two S-wave D mesons. Similar discussions can be easily extended to the B meson system.

- $\bar{D} D^{*}[I=0, J=1]=\mathrm{X}(3872)$

- $\bar{D} D^{*}[I=1, J=1]=Z_{c}(3900)$ multiplet

- $\bar{D}^{*} D^{*}[I=0, J=0,1,2]=\operatorname{missing}$

- $\bar{D}^{*} D^{*}[I=1, J=1]=Z_{c}(4020)$ multiplet

Maybe Belle's $Z^{+}(4051)$ observed in the $\pi \chi_{c 1}^{\prime}$ channel is a member of this multiplet?

- $\bar{D}^{*} D^{*}[I=1, J=0]=$ missing?

- $\bar{D}^{*} D^{*}[I=1, J=2]=$ missing or does not exist? 
- Strange or hidden-strangeness partners?

There are also possible molecules composed of one S-wave D meson and another P-wave D meson.

- $D_{1} \bar{D}[I=0, J=1]=\mathrm{Y}(4260)$

- $D_{1} \bar{D}[I=1, J=1]=$ ?

Maybe Belle's $Z^{+}(4248)$ observed in the $\pi \chi_{c 1}^{\prime}$ channel is a member of this multiplet?

- $D_{1} \bar{D}^{*}[I=0, J=1]=\mathrm{Y}(4360)$ ?

- $D_{1} \bar{D}^{*}[I=0, J=0,2]=$ missing

- $D_{1} \bar{D}^{*}[I=1, J=0,1,2]=$ ?

Maybe Belle's $Z^{+}$(4430) observed in the $J / \psi \pi$ channel is a member of this multiplet [24, $25]$ ?

- $D_{2} \bar{D}[I=0,1 ; J=2]=$ missing or does not exist?

- $D_{2} \bar{D}^{*}[I=0,1 ; J=1,2,3]=$ missing or does not exist?

- Strange or hidden-strangeness partners?

Maybe $\mathrm{Y}(4660)$ is an isoscalar hidden-strangeness member belonging to this group?

\section{Summary}

The excited charmonium or Upsilon states act as a hadron molecule factory. The $Y(5 S)$ mass is $10.86 \mathrm{GeV}$ while $M_{B}+M_{B^{*}}+M_{\pi}=10.744 \mathrm{GeV}$ and $2 M_{B^{*}}+M_{\pi}=10.79 \mathrm{GeV}$. The phase space of the $Y(5 S) \rightarrow B(*) \bar{B}(*) \pi$ decay is tiny. The relative motion between the B meson pair is very slow, which is favorable to the formation of the molecular states. $Y(5 S, 6 S)$ is the ideal factory of molecular states. These molecular states will be produced abundantly at BelleII in the near future!

Similarly, the excited charmonium decay such as $\mathrm{Y}(4260,4360,4660)$ etc is ideal in the search of the D meson molecular states. The $\gamma, 1 \pi, 2 \pi, 3 \pi$ and other light degree of freedom will act as a quantum number filter of these interesting states.

$\mathrm{X}(3872), \chi_{c 1}^{\prime}$ and $\mathrm{Y}(4260)$ are the key states in revealing the underlying structure of the charmonium-like XYZ states. Through the decay pattern and possible partner states, we can test the molecule picture. Especially the experimental measurement of the various pionic and electromagnetic transitions between $\mathrm{Y}(4360), \mathrm{Y}(4260), Z_{c}(4020), Z_{c}(3900)$ and $\mathrm{X}(3872)$ are crucial.

\section{Acknowledgments}

This project was supported by the National Natural Science Foundation of China under Grants 11075004,11021092 , and 11261130311. 


\section{References}

[1] D.Y. Chen, J. He and Xiang Liu, Phys. Rev. D83 (2011) 054021.

[2] B. Q. Li and K. T. Chao, Phys.Rev. D79 (2009) 094004.

[3] W. Chen and Shi-Lin Zhu, Phys. Rev. D83 (2011) 034010.

[4] Shi-Lin Zhu, Phys. Lett. B625 (2005) 212.

[5] E. Kou, O. Pene, Phys. Lett. B631 (2005) 164.

[6] F. E. Close, P. R. Page, Phys. Lett. B628 (2005) 215.

[7] Hadron Spectrum Collaboration (L. Liu et al.), JHEP 1207 (2012) 126.

[8] Shi-Lin Zhu, Phys. Rev. D60 (1999) 014008.

[9] A. Bondar et al. (Belle Collaboration), Phys. Rev. Lett. 108 (2012) 122001.

[10] M. Ablikim et al. (BESIII Collaboration), Phys. Rev. Lett. 110 (2013) 252001.

[11] M. Ablikim et al. (BESIII Collaboration), arXiv:1309.1896.

[12] Z. Q. Liu et al. (Belle Collaboration), Phys. Rev. Lett. 110 (2013) 252002.

[13] T. Xiao, S. Dobbs, A. Tomaradze and K. K. Seth, arXiv:1304.3036 [hep-ex].

[14] Z. F. Sun, J. He, X. Liu, Z. G. Luo, Shi-Lin Zhu, Phys. Rev. D84 (2011) 054002.

[15] X. Liu, Z. G. Luo, Y. R. Liu, Shi-Lin Zhu, Eur. Phys. J. C61 (2009) 411.

[16] Y. R. Liu, X. Liu, W. Z. Deng, Shi-Lin Zhu, Eur. Phys. J. C56 (2008) 63.

[17] J. He, X. Liu, Z. F. Sun, Shi-Lin Zhu, Eur. Phys. J. C73 (2013) 2635.

[18] Z. F. Sun, Z. G. Luo, J. He, X. Liu, Shi-Lin Zhu, Chin. Phys. C36 (2012) 194.

[19] A. E. Bondar, A. Garmash, A. I. Milstein, R. Mizuk, M. B. Voloshin, Phys. Rev. D84 (2011) 054010.

[20] M. B. Voloshin, Phys. Rev. D84 (2011) 031502.

[21] S. Ohkoda, Y. Yamaguchi, S. Yasui, K. Sudoh, A. Hosaka, Phys. Rev. D86 (2012) 014004.

[22] N. Li, Shi-Lin Zhu, Phys. Rev. D86 (2012) 074022.

[23] M. Ablikim et al. (BESIII Collaboration), arXiv:1310.4101v1 [hep-ex].

[24] X. Liu, Y. R. Liu, W. Z. Deng, Shi-Lin Zhu, Phys. Rev. D77 (2008) 034003.

[25] X. Liu, Y. R. Liu, W. Z. Deng, Shi-Lin Zhu, Phys. Rev. D77 (2008) 094015. 